\title{
Synthesis and Characterization of Carbon Nanotubes Decorated with Gold Nanoparticles
}

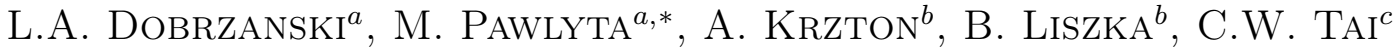 \\ AND W. KWASNY ${ }^{a}$ \\ ${ }^{a}$ Institute of Engineering Materials and Biomaterials, Silesian University of Technology \\ Konarskiego 18A, 44-100 Gliwice, Poland \\ ${ }^{b}$ Centre of Polymer and Carbon Materials, Polish Academy of Sciences \\ Sowinskiego 5, 44-121 Gliwice, Poland \\ ${ }^{c}$ Department of Materials and Environmental Chemistry, Arrhenius Laboratory \\ Stockholm University, SE-106 91 Stockholm, Sweden
}

\begin{abstract}
In presented work we report results of simple and viable method for producing Au/CNT composites. Chemical composition and crystallographic structure of the Au/CNT composites was confirmed by X-ray diffraction measurements, while transmission and scanning electron microscopy were used to characterize the morphology of nanocrystals as well as the distribution of nanocrystals in the composite. The obtained particles with relatively small diameter (less than $9 \mathrm{~nm}$ ) were found to be spatially well dispersed on the carbon nanotubes. The density of attached $\mathrm{Au}$-nanoparticles is not sufficient, and cannot be improved by simple increasing gold loading.
\end{abstract}

PACS numbers: 81.07.Nb

\section{Introduction}

Nanocomposites of carbon nanotubes (CNT) and nanoparticles (NPs) in one discrete structure enable to combine the unique physical and chemical properties of these two kinds of materials, leading to novel and prospective applications [1]. There is a great deal of interest in attaching noble metal nanoparticles to nanotube surface due to its potential application in sensors, optical electronics, and heterogeneous catalysis [2-7]. Because of diverse set of applications, different kinds of electrochemical, chemical and physical methods have been developed to synthesize these nanocomposites [8]. Chemical reduction deposition protocol involves the adsorption of a metal salt onto the CNT surface followed by reduction of the metal salt in the presence of reducing agents (for instance $\mathrm{NaBH}_{4}$, citric acid, or ethylene glycol). The main obstacle in efficiently obtaining carbon nanotubenanoparticle composites results from the chemically stable and highly hydrophobic nature of the sidewall of carbon nanotubes. For that reason surface modification is an essential step in any applied treatment. Simple approach reported by Shi et al. [9] by using a small amount of ethanol to reduce the interfacial tension between CNTs and water, thereby modifying their wettability can be used to directly attach gold nanoparticles on carbon nanotubes with high dispersivity. In presented work we report results of synthesis of carbon nanotubes decorated

* corresponding author; e-mail: miroslawa.pawlyta@polsl.pl with gold nanoparticles by that method. Chemical composition and crystallographic structure of the $\mathrm{Au} / \mathrm{CNT}$ composites was confirmed by X-ray diffraction (XRD) measurements, while transmission (TEM) and scanning electron microscopy (SEM) were used to characterize the morphology of nanocrystals as well as the distribution of nanocrystals in the composite.

\section{Experimental}

\subsection{Synthesis of CNT}

To prepare the carbon nanotubes, a quartz boat with cobalt catalysts was placed in a chemical vapor deposition (CVD) reactor. The cobalt catalyst was prepared according to the method reported by Chen et al. [10] and calcined at $795 \mathrm{~K}$ in air for $2 \mathrm{~h}$ and reduced in hydrogen atmosphere for $2 \mathrm{~h}$ [11]. The reactor temperature was increased under an argon flow to $795 \mathrm{~K}$ and then kept for 15 min in hydrogen atmosphere (5\% in argon balance). Then the feed gas $\left(20 \% \mathrm{CO}\right.$ and $5 \% \mathrm{H}_{2}$ in ratio 1:1) was initiated with flow rate at $45 \mathrm{ml} / \mathrm{min}$. The process was terminated after $2 \mathrm{~h}$, and the furnace was turned off and allowed to cool to room temperature.

\subsection{The prior synthesis of $A u-N P s$}

$\mathrm{Au}-\mathrm{NPs}$ were prepared by the method reported by Jana et al. [12]. Firstly $0.5 \mathrm{~mL}$ of $0.01 \mathrm{M} \mathrm{HAuCl}_{4} \cdot 3 \mathrm{H}_{2} \mathrm{O}$ aqueous solution and $0.5 \mathrm{~mL}$ of an aqueous $0.01 \mathrm{M}$ trisodium citrate solution was mixed with $18.4 \mathrm{~mL}$ of deionized (DI) water. Then $0.6 \mathrm{~mL}$ of $0.1 \mathrm{M} \mathrm{NaBH}_{4}$ solution was added while intensive stirring. The color 
of the resultant mixture changed, indicating the formation of $\mathrm{Au}-\mathrm{NPs}$. In such method, $\mathrm{AuCl}_{4}^{-}$was reduced to metallic gold, $\mathrm{BH}_{4}^{+}$was the source of electrons while citrate controls the particle growth.

\subsection{Au/CNT nanocomposites synthesis with different loading ratio of gold}

$\mathrm{Au} / \mathrm{CNT}$ nanocomposites were prepared by the method reported by Shi et al. [9]. The measured amount of hydrophobic CNTs was immersed into the as-prepared gold suspension $(10 \mathrm{~mL}$ ) and mixed with ethanol (about $5 \mathrm{~mL}$ ). A small amount of ethanol can reduce the interfacial tension between CNTs and water, thereby enhancing their wettability in aqueous solution. After ultrasonic dispersion and subsequent stirring for several hours for $8 \mathrm{~h}$ the black solid was separated and washed with DI water for several times and dried for $8 \mathrm{~h}$ in an oven at $70^{\circ} \mathrm{C}$. The amount of added CNTs was determined in order to obtain different composition with different weight percentage (wt\%) of gold: 5, 10, 30, 50, 80 and $100 \mathrm{wt} \%$.

\subsection{Characterization of obtained $A u / C N T$ composites}

The samples with different gold loading ratio were characterized by powder XRD (Panalytical X'Pert Pro, Co $K_{\alpha}$ radiation, $\left.\lambda=0.1789 \mathrm{~nm}\right)$. SEM images in $\mathrm{SE}$ mode were taken with a Zeiss Supra 35 field emission SEM equipped with energy dispersive X-ray (EDX) analyzer. Samples for transmission electron microscopy were prepared by dispersing powder in ethanol, placing in an ultrasonic bath, then putting droplets onto $3 \mathrm{~mm}$ copper grids coated with amorphous carbon film and drying in air at room temperature. High-resolution TEM (HRTEM) images were obtained using a $200 \mathrm{kV}$ JEOL JEM-2100F transmission electron microscope, equipped with a field emission gun and EDX analyzer.

\section{Results and discussion}

The XRD parents for the samples with different wt\% loading ratio of gold are shown in Fig. 1. The diffraction peaks in each XRD pattern at 45.0, 52.5, 77.1, 93.8 and $99.4^{\circ}$ can be assigned to reflections from the (111), (200),

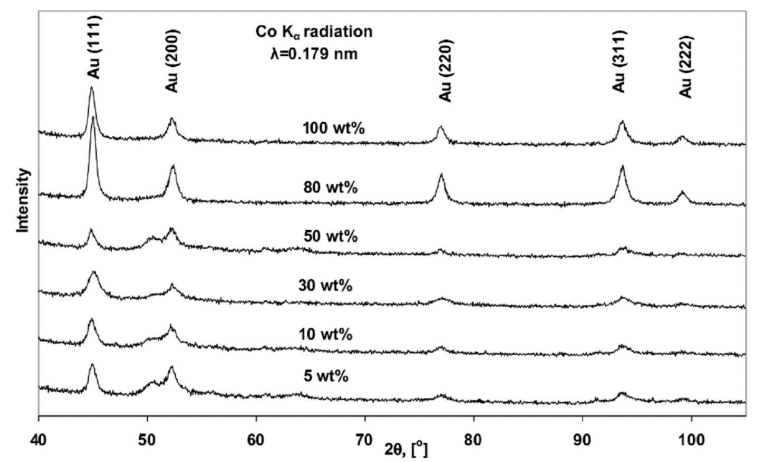

Fig. 1. The XRD patterns of the samples with different gold wt\% loading ratio.
(220), (311) and (222) planes of the face-centered-cubic $\mathrm{Au}$. The broad peaks in the XRD pattern indicate that the gold nanoparticles are small. The average crystallite size of Au was calculated using the Debye-Scherrer formula

$$
d=\frac{K \lambda}{\beta \cos (\theta)},
$$

in which $d$ is the crystallite size, $\lambda$ - the wavelength of the X-ray radiation, $K$ is usually taken as $0.89,2 \theta$ is the Bragg angle of the $\mathrm{Au}$ (111) peak, and $\beta$ is the line width at half-maximum height, after subtraction of equipment broadening. The average crystallite size of gold nanoparticles was determined to be between 4 and $9 \mathrm{~nm}$, but no significant dependence on gold loading ratio was observed. The small peak at $51.8^{\circ}$ in XRD pattern for 5 , 10 , and $50 \mathrm{wt} \%$ can be attributed to remain impurities from catalyst (reflection from the (111) planes of Co).

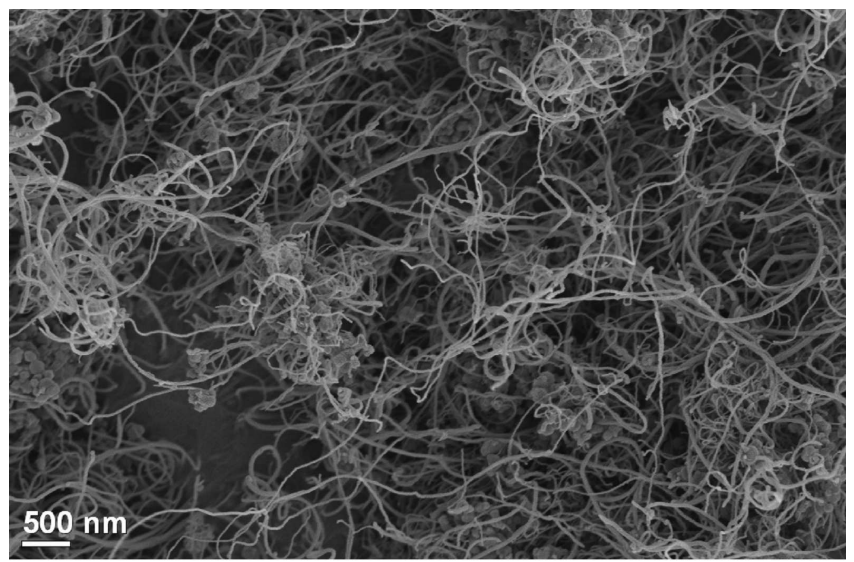

Fig. 2. SEM image of pure CNT.

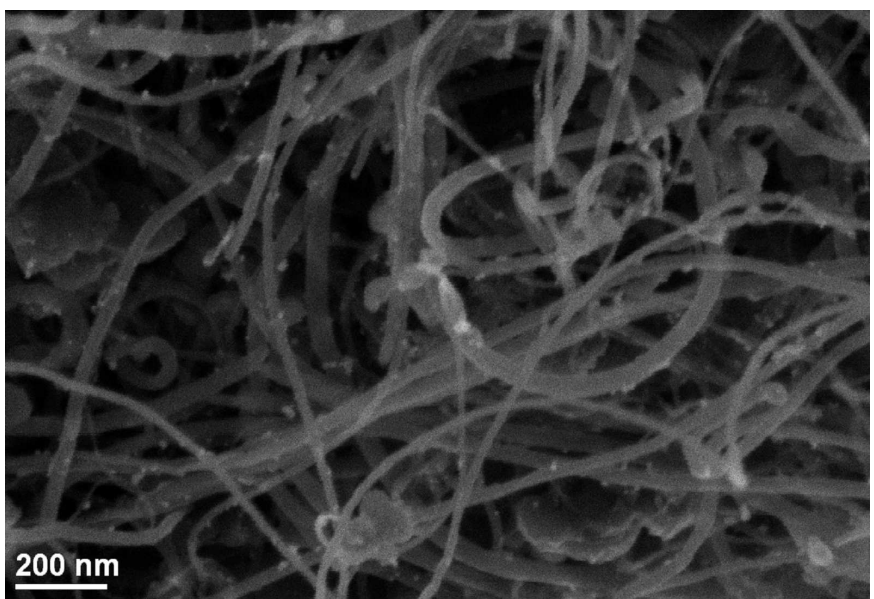

Fig. 3. SEM image of CNT with 5 wt\% gold loading ratio.

Figure 2 shows a SEM image in SE mode of the as prepared CNTs in carbon monoxide disproportional reaction from $\mathrm{Co} / \mathrm{MgO}$ catalyst. The obtained carbon nanotubes 


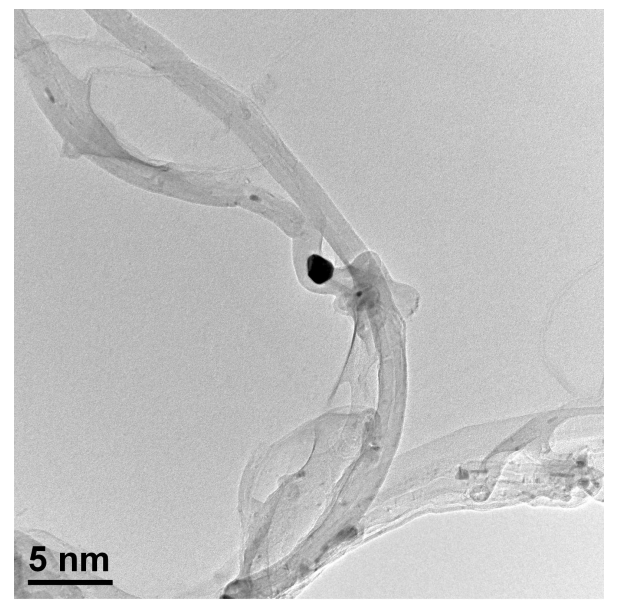

Fig. 4. TEM images - BF image of a single CNT with dark contrast corresponding to gold nanoparticles (CNT with 5 wt $\%$ gold loading ratio).

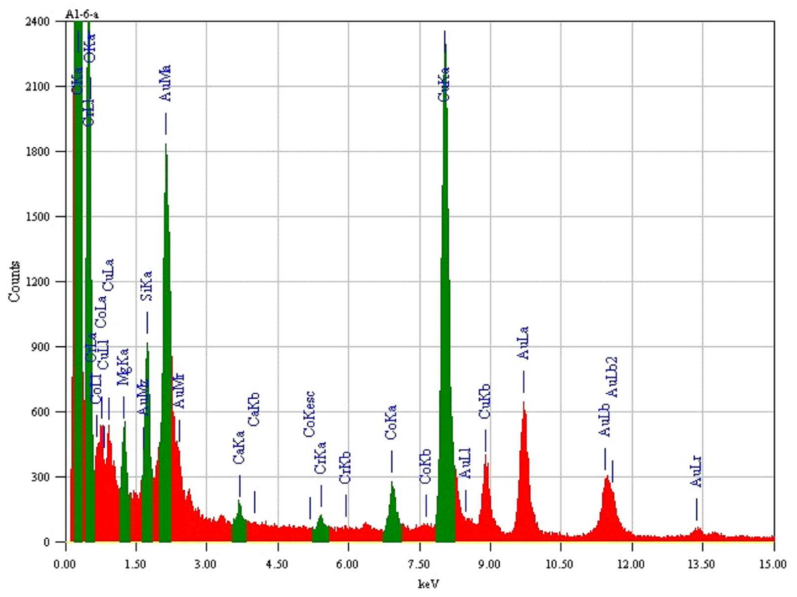

Fig. 5. EDX spectrum of $\mathrm{Au} / \mathrm{CNT}$ composites imaged in Fig. 4.

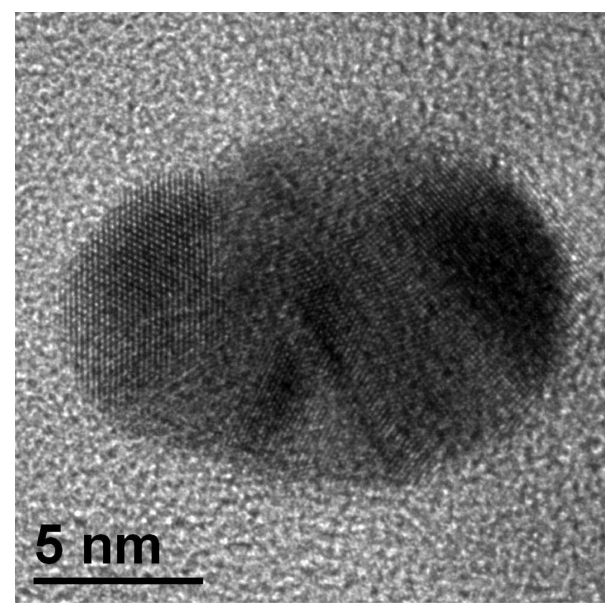

Fig. 6. HRTEM images of Au nanoparticle.

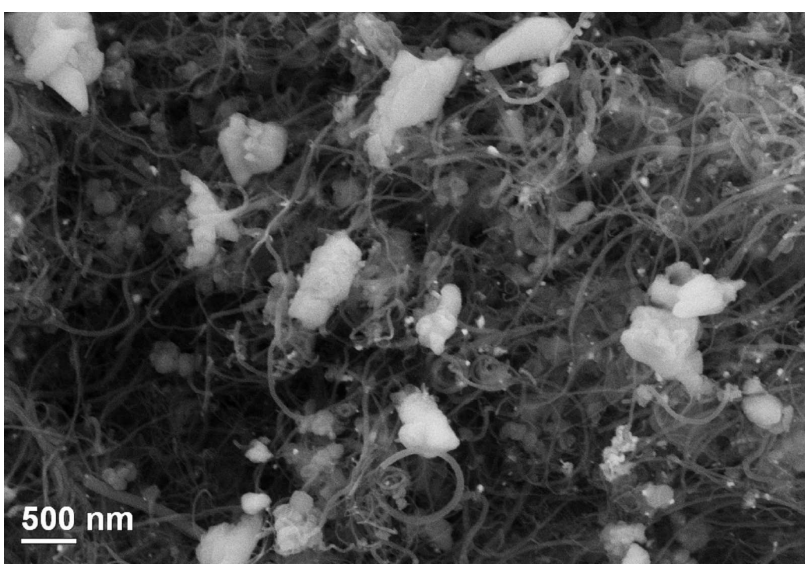

Fig. 7. SEM image of CNT with $100 \mathrm{wt} \%$ gold loading ratio.

are formed as a network with different densities and contain large amount of remained impurities (catalysts), visible as spherical shapes. CNT diameters can be estimated as between 10 and $120 \mathrm{~nm}$ and length up to $10 \mu \mathrm{m}$.

Figure 3 shows a SEM image in SE mode of the $\mathrm{Au} / \mathrm{CNT}$ composites with $5 \mathrm{wt} \%$ loading ratio of $\mathrm{Au}$, where the $\mathrm{Au}-\mathrm{NPs}$ are seen as bright spots on the CNT surfaces, fairly homogeneously distributed. The Au phase is present in relatively small particles, the size of the largest being consistent with X-ray results.

Representative TEM image of the nanocomposites is presented in Fig. 4. The observed particles appear to have a narrow size distribution, and no free particles were observed in the background of the TEM images, which confirms that all formed $\mathrm{Au}-\mathrm{NPs}$ were attached to the nanotubes.

In Fig. 5 the EDX spectrum of $\mathrm{CNT} / \mathrm{Au}$ nanocomposites reveals the presence of $\mathrm{Au}$. The peaks of Co and $\mathrm{Mg}$ elements are from the catalysts while the peak of $\mathrm{O}$ element is probably from the sodium citrate. The copper peak could be attributed to the copper of the microscopy grid. The particle sizes determined from TEM are in close agreement with those obtained from XRD, indicating a good crystallinity of the supported gold nanoparticles.

Crystallographic structure of gold particle is well visible on a HRTEM image (Fig. 6).

While no obvious aggregation was observed in the $5 \mathrm{wt} \% \mathrm{Au}$ content sample, many larger in size aggregates of gold particles can be found in the $100 \mathrm{wt} \% \mathrm{Au}$ content sample (Fig. 7).

\section{Conclusions}

Chemical reduction deposition protocols of gold nanoparticles (NPs) onto carbon nanotubes (CNTs) was demonstrated in this work. Simple and effective method include the adsorption of $\mathrm{Au}$ nanoparticles onto the CNT surface followed by reduction of the metal salt in the presence of citric acid, while ethanol addition reduces 
the interfacial tension between CNTs and aqueous solution containing $\mathrm{Au}-\mathrm{NPs}$. The particles with relatively small diameter (less than $9 \mathrm{~nm}$ ) were found to be spatially well dispersed on the carbon nanotubes. The density of attached $\mathrm{Au}-\mathrm{NPs}$ is not sufficient, and cannot be improved by simple increasing gold loading due to aggregation. This work demonstrates a simple and viable method for producing $\mathrm{Au} / \mathrm{CNT}$ composites for applications in a variety of areas including catalysis, fuel cells, and electrochemical sensors. This preparation method is expected to be also applied to the synthesis of platinum/ CNT and silver/CNT hybrid composites.

\section{Acknowledgments}

The Knut and Alice Wallenberg Foundation is acknowledged for an equipment grant for the electron microscopy facilities in Stockholm University.

\section{References}

[1] X. Peng, J. Chen, J.A. Misewich, S.S. Wong, Chem. Soc. Rev. 38, 1076 (2009).

[2] S.W. Lee, W.M. Sigmund, Chem. Commun. 6, 780 (2003).
[3] G. Yamamoto, M. Omori, K. Yokomizo, T. Hashida, K. Adachi, Mater. Sci. Eng. B 148, 265 (2008).

[4] P. Santhosh, A. Gopalan, K.P. Lee, J. Catal. 238, 177 (2006).

[5] J.T. Abrahamson, N. Nair, M.S. Strano, Nanotechnology 19, 195701 (2008).

[6] J. Kong, M.G. Chapline, H.J. Dai, Adv. Mater. 13, 1384 (2001).

[7] R. Meyer, C. Lemire, Sh.K. Shaikhutdinov, H.J. Freund, Gold Bull 37, 72 (2004).

[8] G.G. Wildgoose, C.E. Banks, R.G. Compton, Small 2, 182 (2006).

[9] Y. Shi, R. Yang, P.K. Yuet, Carbon 47, 1146 (2009).

[10] P. Chen, H.B. Zhang, G.D. Lin, Q. Hong, K.R. Tsai, Carbon 35, 1495 (1997).

[11] J.P. Pinheiro, M.C. Schouler, P. Gadelle, Carbon 41, 2949 (2003).

[12] N.R. Jana, L. Gearheart, C.J. Murphy, Langmuir 17, 6782 (2001). 